\title{
ANÁLISE DA PRODUÇÃO DO CONHECIMENTO EM PROCESSO DE ENFERMAGEM: estudo exploratório-descritivo
}

\author{
E rika Christiane M arocco DURAN ${ }^{a}$, Vanessa Pellegrino T OLE D O ${ }^{a}$
}

\section{RESUM 0}

Estudo descritivo-exploratório que objetiva analisar a produção do conhecimento em processo de enfermagem com base nas dissertações de mestrado e teses de doutorado defendidas nos prog ramas de pós-graduação em enfermagem do Brasil, por meio dos relatórios do Centro de Estudos e Pesquisas em Enfermagem (CEPEn) de 1972 a 2007 e diagnosticar as publicações em bases de dados indexadas. Encontrou-se 122 dissertações de mestrado, com 42 publicações, 26 teses de doutorado, com 15 publicações. Verificou-se maior publicação a partir de 2000, sendo a pesquisa qualitativa mais prevalente. A tendência temática mais evidenciada foi assistência de enfermagem, sendo o levantamento e validação de diagnóstico de enfermagem etodas as fases do processo os assuntos mais abordados. Existem lacunas nas publicações sobre o tema, sobretudo no levantamento da produção do conhecimento. Pesquisas que analisem esta interface possivelmente podem qualificar a implementação do processo de enfermagem.

D escritores: Pesquisa em enfer magem. Assistência de enfer magem. Publicações periódicas.

\section{RESUMEN}

E studio descriptivo-exploratorio con objeto de analizar la producción de conocimiento sobre el proceso de enfermería basado en las disertaciones de maestría y tesis de doctorado defendidas en los programas de postgrado en enfermería de B rasil, mediante los infor mes del Centro de E studios e Investigaciones en E nfermería (CE PE n) entre 1972 y 2007 y diagnosticar Ias publicaciones en bases de datos indexadas. F ueron encontradas 122 disertaciones de maestría, con 42 publicaciones, y 26 tesis de doctorado, con 15 publicaciones. Se verificó mayor publicación a partir de 2000, siendo la investigación cualitativa más prevalente $L$ a tendencia temática más evidenciada fue el asistencia de enfermería, siendo el análisis y la validación de diagnóstico de enfermería y todas las fases del proceso los tópicos más tratados. Existen lagunas en las publicaciones sobre el tema, sobretodo en el análisis de la producción de conocimiento. Investigaciones que analicen este interfaz posi blemente puede cualificar la implementación del proceso de enfermería.

D escriptores: Investigación en enfermería. A tención de enfermería. P ublicaciones periódicas.

T ítulo: A nálisis de la producción de conocimiento sobre el proceso de enfermería: estudio exploratorio-descriptivo.

\section{ABST RACT}

This descriptive exploratory study aims to analyze the production of knowledge on the nursing process, based on M aster's theses and doctoral dissertations presented in B razilian graduate programs in N ursing, using the reports of the N ursing Study and Research Center (CE PE n) from 1972 to 2007, and to identify which were published in indexed databases. We found $122 \mathrm{M}$ aster's theses, 42 of which were published, and $26 \mathrm{D}$ octoral dissertations, with 15 publ ications. F rom the year 2000 on more publications w ere found, with a prevalence of qualitative research. T he prevalent thematic trend was nursing assi stance, with surveys and validation of nursing diagnosis, as well as the other phases of the process, as the most addressed topics. Publications on thethemeshow gaps, especially in surveying know ledge production. R esearches that study this interface may possibly qualify the practice of nursing.

Descriptors: N ursing research. N ursing care. Periodicals.

T itle: Analysis of knowledge production on the nursing process: explorator y descriptive study.

\footnotetext{
a D outora em Enfermagem, Professora D outora do D epartamento de Enfermagem da Faculdade de Ciências M édicas da Universidade Estadual de Campinas (U N ICAM P), Campinas, São Paulo, Brasil.
} 


\section{INT RODUÇÃO}

0 processo de enfermagem (PE) pode ser definido como um método dinâmico, flexível, organizado e utilizado na prática clínica da enfermagem para orientar o trabal ho do enfermeiro na investigação dos dados do paciente, identificando as necessidades de cuidados, propondo intervenções e avaliando os resultados dos cuidados que real iza, que inclui a sistematização da assistência de enfermagem (SAE) como el emento de organização e operacionalização. Considera-se que a documentação do PE é um instrumento útil para a avaliação do cuidado através do gerenciamento das infor mações de enfer magem ${ }^{(1)}$.

Entende-se que o processo de trabalho do enfermeiro inclui o gerenciamento do cuidado e que 0 processo de enfermagem consiste em um instrumento desse trabal ho que pode qualificar a assistência de enfer magem, desde que sua aplicação esteja pautada em uma apreensão ampliada das necessidades de cuidado dos usuários e orientada na perspectiva do cuidado integral ${ }^{(2)}$.

A primeira publicação sobre o PE no Brasil foi realizada em 1979 por W anda H orta com o livro intitulado "O Processo de Enfermagem"(3). A partir deste documento e da utilização do PE na prática dos enfermeiros, constituindo o corpo de conhecimento próprio da enfermagem, as publicações sofreram incremento gradativo, tanto no ensino, pesquisa, assistência e gerenciamento.

0 conjunto da produção constante se constitui em uma contribuição à leitura e à reflexão, além de oferecer subsídios para a atuação em Enfermagem ${ }^{(4)}$.

A média da produção em 2006 , em pesquisa objetivando caracterizar a produção científica nacional sobre o termo SAE e visualizar tendências da mesma entre 1990 e 2002, foi de 13,4 publicações/ ano e os artigos distribuídos em 23 periódicos com predomínio de estudos realizados na área hospitalar. Q uanto à temática, 17,6\% focavam a cardiologia, 78,6\% a implementação, desenvolvimento e avaliação dos modelos de SAE. A taxonomia $\mathrm{N}$ orth American $\mathrm{N}$ ursing $\mathrm{D}$ iagnosis $\mathrm{A}$ ssociation (NA N D A) foi citada em 40,2\% e W anda H orta em $40,5 \%$ das publicações ${ }^{(5)}$.

A análise da produção do conhecimento em PE evidencia as temáticas abordadas e seus focos, 0 desenho das pesquisas, contribui para a identificação de lacunas e construção de um processo en- sino-aprendizagem fidedigno no que se refere à organização de conteúdo de ensino, corroborando com uma práxis integradora do docente-pesquisador.

Dessa forma, a presente pesquisa objetiva analisar a produção do conhecimento em processo de enfermagem com base nas dissertações de mestrado e teses de doutorado defendidas nos programas de pós-graduação em enfermagem do Brasil no período de 1972 a 2007 e o seu diagnóstico no que tange à divulgação do conhecimento em periódicos científicos nacionais e internacionais indexados, caracterizando-a segundo o objeto de estudo, as abordagens metodológicas e ano de produção.

\section{MÉTODOS}

Pesquisa descritivo-exploratória pela característica de observar, classificar e descrever fenômenos efetuada através dos relatórios do Centro de Estudos e Pesquisas em Enfermagem (CEPEn) da Associação Brasileira de Enfermagem (ABEN) de 1972 a 2007. A escolha do início do período desta investigação deve-se ao fato da existência das primeiras produções de enfermagem. A pós a identificação da publicação foi realizada a leitura dos resumos das dissertações e teses e registrados dados de identificação da produção (segundo a autoria, objeto de estudo, abordagens metodológicas e ano de produção). A pós, realizou-se o levantamento dos artigos oriundos destas publicações nas bases de dados: Literatura L atino-A mericana e do Caribe em Ciências da Saúde (LILACS), M edical Literature Analysis and Retrieval System O nline (M EDLINE) e PubM ed, utilizando o sobrenome dos autores. A análise do delineamento metodológico das pesquisas foi realizada com base nas categorias de estudos quantitativos (experimental, quase-experimental e não-experimental) e qualitativos (etnografia, fenomenologia e teoria fundamentada)(6).

Utilizou-se a planilha do programa computacional Excel para a inserção dos dados. A análise foi descritiva-qual itativa e quantitativa.

\section{RESULT ADOS}

Os resultados mostram que no período estudado foram defendidas 122 dissertações de mestrado e 26 teses de doutorado na temática estudada nos Programas de Pós-Graduação em Enfermagem. 
T abela 1 - D istribuição das dissertações de mestrado e teses de doutorado em processo de enfermagem segundo 0 ano de conclusão, 1972-2007.

\begin{tabular}{cccc}
\hline \multirow{2}{*}{ Ano de conclusão } & Tese de D outorado & Dissertação de M estrado & T otal \\
\cline { 2 - 4 } & $\mathbf{n}$ & $\mathbf{n}$ & $\mathbf{n}$ \\
\hline 1975 & 1 & - & 1 \\
1981 & 1 & - & 1 \\
1989 & 2 & - & 2 \\
1990 & 2 & - & 2 \\
1991 & 4 & - & 4 \\
1992 & 2 & 1 & 3 \\
1993 & 2 & 1 & 3 \\
1994 & 2 & - & 2 \\
1995 & - & 2 & 2 \\
1996 & 4 & - & 4 \\
1997 & 7 & 1 & 8 \\
1998 & 5 & - & 5 \\
1999 & 8 & 2 & 10 \\
2000 & 20 & 4 & 24 \\
2001 & 15 & - & 15 \\
2002 & 6 & 2 & 8 \\
2003 & 10 & 3 & 13 \\
2004 & 5 & 4 & 9 \\
2005 & 10 & 4 & 7 \\
2006 & 5 & 2 & 11 \\
2007 & 11 & - & $\mathbf{1 4 8}$ \\
\hline T otal & $\mathbf{1 2 2}$ & $\mathbf{2 6}$ & \\
\hline
\end{tabular}

A T abela 1 apresenta a distribuição das dissertações de mestrado e teses de doutorado no período investigado. Observa-se que a partir de 2000 houve um incremento na produção do conhecimento em processo de enfermagem.

$\mathrm{Na}$ T abela 2 constata-se 0 delineamento metodológico das pesquisas. Evidencia-se nas dissertações de mestrado a pesquisa qual itativa etnográfica (31,97\%), fenomenológica $(30,35 \%)$ e teo- ria fundamentada $(19,67 \%)$ como delineamentos metodológicos mais utilizados. $\mathrm{N}$ as teses de doutorado, a pesquisa quantitativa não experimental (26, 93\%) e a qual itativa fenomenológica (26,93\%), seguidas pela qualitativa teoria fundamentada $(19,23 \%)$ e etnog ráfica (19,23\%). D estaca-se que em ambos os casos a pesquisa quantitativa experimental foi a menos utilizada.

T abela 2 - Distribuição das dissertações de mestrado e teses de doutorado produzidas nos Programas de Pós-G raduação em E nfermagem segundo o delineamento metodológico das pesquisas, 1972-2007.

\begin{tabular}{lccc}
\hline T ipos de pesquisa & T ese de D outorado & Dissertação de M estrado & T otal \\
\cline { 2 - 5 } & $\mathbf{n}$ & $\mathbf{n}$ & $\mathbf{n}$ \\
\hline Quantitativa Experimental & 1 & - & 2 \\
Quantitativa Quase-experimental & 6 & 7 & 6 \\
Quantitativa N ão experimental & 9 & 7 & 16 \\
Qualitativa / Fenomenologia & 37 & 5 & 44 \\
Qualitativa / T eoria Fundamentada & 24 & 5 & 29 \\
Qualitativa / E tnog rafia & 39 & - & 44 \\
Quali-quantitativa / E tnografia & 4 & & 4 \\
e Na âo-experimental & & 1 & 3 \\
Quali-quantitativa / Fenomenologia & 2 & $\mathbf{2 6}$ & $\mathbf{1 4 8}$ \\
e Não-experimental & $\mathbf{1 2 2}$ & &
\end{tabular}


Q uanto às tendências temáticas, observa-se na T abela 3 um incremento das pesquisas no assunto assistência de enfermagem, sendo que $32,78 \%$ se destinaram à levantamento de diagnóstico de enfermagem, $19,67 \%$ à todas as fases do processo e $16,39 \%$ à validação de diagnostico de enfermagem. No assunto ensino do processo de enferma- gem, uma pesquisa evidenciou o levantamento de diagnóstico e o processo ensino-aprendizagem. $\mathrm{Na}$ revisão sistemática, verificou-se uma pesquisa sobre feridas crônicas e outra sobre características definidoras e fatores relacionados do diagnóstico de náusea.

T abela 3 - Distribuição das dissertações de mestrado segundo a tendência temática dos estudos, 19722007.

\begin{tabular}{|c|c|}
\hline T endências temáticas das pesquisas & $\mathrm{n}$ \\
\hline $\begin{array}{l}\text { 1. Processo de Enfermagem } \\
\text { Todas as fases } \\
\text { Histórico de Enfer magem } \\
\text { H istórico e D iagnóstico de E nfer magem } \\
\text { Histórico, D iagnóstico e Planejamento de E nfermagem } \\
\text { H istórico e Planejamento de E nfer magem } \\
\text { Histórico, Planejamento e Avaliação de E nfer magem } \\
\text { H istórico, Planejamento, I mplementação e Avaliação } \\
\text { Diagnóstico e Planejamento de E nfer magem } \\
\text { Diagnóstico, P lanejamento e Avaliação de E nfer magem } \\
\text { L evantamento de Diagnóstico de Enfer magem } \\
\text { Validação de D iagnóstico de E nfer magem } \\
\text { Planejamento de E nfermagem } \\
\text { Implementação de Enfermagem } \\
\text { Planejamento e I mplementação de E nfer magem }\end{array}$ & $\begin{array}{r}24 \\
7 \\
2 \\
3 \\
1 \\
1 \\
3 \\
6 \\
1 \\
40 \\
20 \\
1 \\
2 \\
1\end{array}$ \\
\hline $\begin{array}{l}\text { 2. Ensino do Processo de E nfermagem } \\
\text { Ensino } \\
\text { L evantamento de D iagnóstico de Enfermagem e Ensino } \\
\text { 3. Revisão sistemática em Processo de Enfermagem }\end{array}$ & $\begin{array}{l}7 \\
1\end{array}$ \\
\hline $\begin{array}{l}\text { Feridas Crônicas } \\
\text { Características definidoras e fatores relacionados do diagnóstico de náusea }\end{array}$ & $\begin{array}{l}1 \\
1\end{array}$ \\
\hline$\overline{\text { T otal }}$ & 122 \\
\hline
\end{tabular}

A T abela 4 apresenta as tendências temáticas nas teses de doutorado. A validação de diagnóstico de enfermagem foi mais freqüente $(23,02 \%)$, seguida pelo estudo de todas as fases do processo de enfermagem (19,23\%). Ressalta-se que não se evidenciou pesquisa no assunto revisão sistemática.

A cerca do diagnóstico da produção na divulgação do conhecimento em periódicos nacionais e inter nacionais destaca-se que as descobertas científicas gal gam reconhecimento e credibilidade diante de sua publicação em revistas de impacto junto a comunidade acadêmica, buscou-se identificar a publicação de artigos extraídos das dissertações de mestrado e teses de doutorado ${ }^{(7)}$.

A busca resultou em 57 artigos, sendo que dissertações de mestrado originaram $42(65,63 \%)$ artigos e as teses de doutorado 15 (26,31\%), perfazendo $38,51 \%$ do total de estudos produzidos no período. O bservou-se que das publicações do mestrado seis $(14,29 \%)$ estavam indexadas na base de dados M ED LINE, 31 (78,80\%) na LILACS e cinco $(6,91 \%)$ PubM ed. Ressalta-se que no ano de 2003, a mesma publicação estava indexada na M ED LINE eLILACS; em 2004, uma pesquisa originou duas publicações indexadas no M EDLINE e em 2005, a mesma publicação estava indexada no PubM ed e MEDLINE.

Constatou-se que, de 2000 a 2007, houve um aumento das publicações referentes ao assunto nas três bases de dados estudadas.

D as publicações do doutorado, oito (40\%) estavam indexadas na base de dados M E D L INE, sete $(35 \%)$ na LILACS e cinco Pubmed (25\%), totalizando 20 devido à duplicação dos artigos nas bases de dados. Ressalta-se que no ano de 2002, a mesma publicação estava indexada na PubM ed e 
T abela 4 - D istribuição das teses de doutorado segundo a tendência temática dos estudos, 1972-2007.

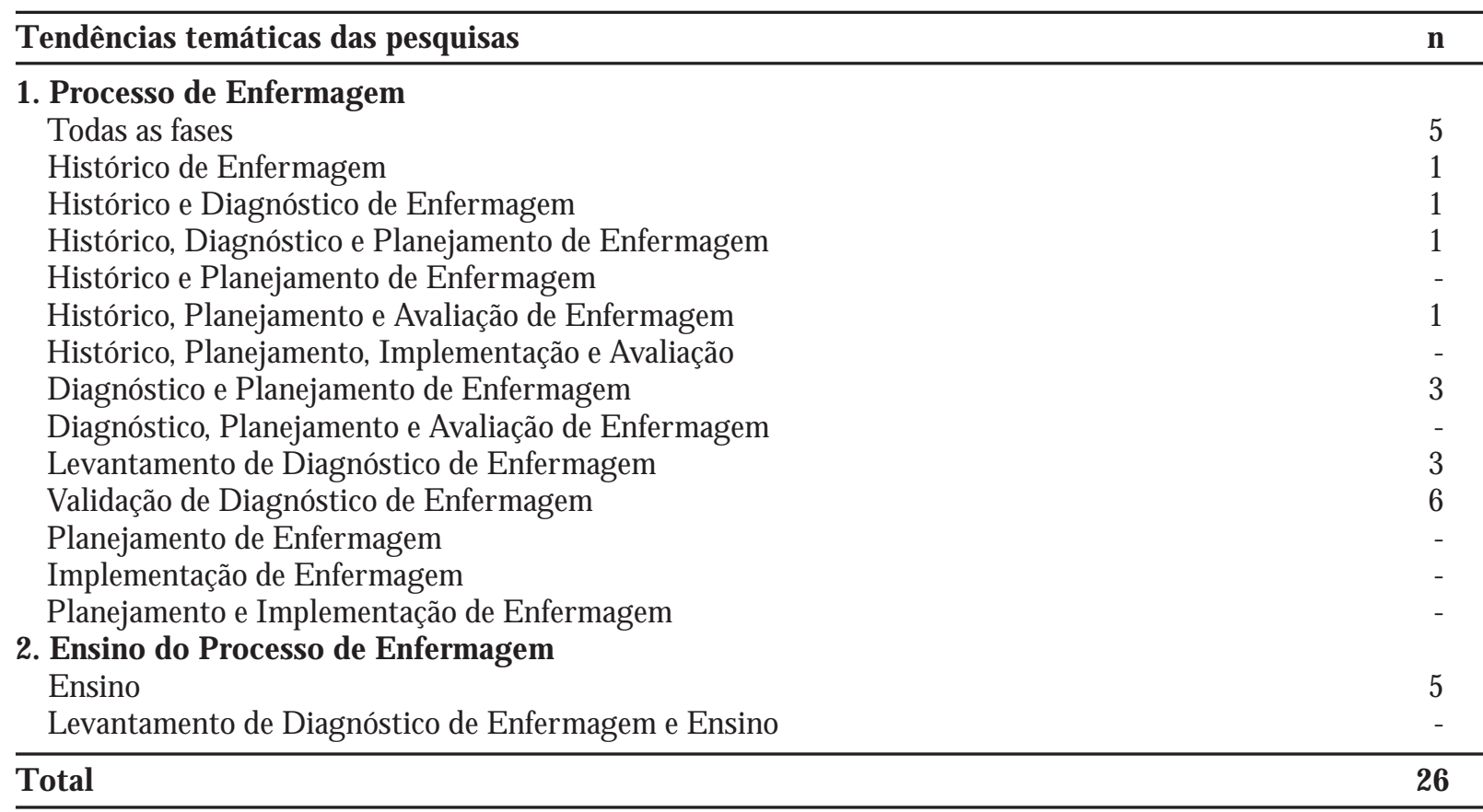

LILACS; em 2004, uma publicação estava indexada naM EDLINE eLILACS, outranaMEDLINE e PubM ed e outra com dois artigos PubM ed; em 2005, uma publicação indexada na M EDLINE e PubM ed. Constata-se que 0 aumento das publicações ocorreu a partir de 2003 e após 2005 não houve mais publicações.

Q uanto às tendências temáticas e delineamento metodológico, as publicações oriundas das dissertações de mestrado apresentaram 20 de levantamento dos diagnósticos de enfermagem, sendo a maioria (13,65\%) qual itativa etnog ráfica; seis de validação de diagnóstico de enfermagem, sendo duas qual itativa e etnográfica, dois fenomenologia e dois quantitativo quase-experimental. $\mathrm{N}$ as publicações oriundas das teses de doutorado obteve-se quatro de validação de diagnóstico de enfermagem, sendo dois qualitativo e teoria fundamentada, uma etnográfica e uma fenomenológica. Na categoria ensino do processo de enfermagem, dois qualitativo e fenomenologia e uma teoria fundamentada. Quanto ao tema todas as fases do processo de enfermagem, evidenciou-se dois qualitativo e etnográfico e um quantitativo não experimental.

\section{DISCUSSÃO}

Desde 21 de janeiro de 2000, a Sistematização da Assistência de Enfermagem tornou-se obri- gatória em todo o estado de São Paulo, de acordo com o determinado pela decisão do Consel ho Regional de Enfermagem - COREN-SP/DIR/ 008/ 99. Este documento incorpora o exercício profissional do enfermeiro, responsável pela implantação, planejamento, organização, execução e aval iação do processo de enfermagem. A fiscalização do CORE N SP continuará a priorizar, em seus trabal hos junto aos enfermeiros, o efetivo cumprimento da determinação ético-profissional, iniciando o resgate do verdadeiro papel que compete ao enfermeiro assu$\mathrm{mir}^{(8)}$.

E $m$ 2002, por meio da resolução do Consel ho Federal de Enfermagem (COFEN) n² 272/ 2002, houve a exigência legal da implantação do processo de enfermagem em todas as unidades de saúde ${ }^{(9)}$.

A pesquisa apresentou um incremento na produção de conhecimento de dissertações de mestrado e teses de doutorado e publicação de artigos a partir de 2000. Os achados corroboram com 0 estudo que infere que este aumento pode estar atrelado à legislação(10).

Os dados da T abela 2 evidenciam que nas dissertações de mestrado o delineamento metodológico qualitativo mais freqüente foi etnografia, fenomenologia e teoria fundamentada e nas teses de doutorado, quantitativa não experimental e qualitativa fenomenológica, seguidas de qual itativa etnográfica e teoria fundamentada. 
Vale ressaltar que a realização dos estudos com delineamento metodológico qualitativo tem sido amplamente utilizada na enfermagem em diversas áreas do conhecimento(11). Autores identificaram que $50 \%$ das pesquisas publicadas no período de 1977 a 2001 apresentaram delineamento qualitativo, sendo a fenomenologia o mais utilizado pelos pesquisadores ${ }^{(12)}$.

$\mathrm{Na}$ pesquisa experimental o pesquisador é um agente ativo, mais do que um observador passivo. Os experimentos não ocorrem necessariamente em laboratórios, podendo ser conduzidos em qualquer ambiente. A manipulação, o controle e a randomização são características do delineamento de pesquisa experimental. $\mathrm{Na}$ pesquisa quaseexperimental há o envolvimento da manipulação, porém não possui características de randomização ou de grupo controle, ou ambas. $\mathrm{N}$ a pesquisa não-experimental a (não) manipulação da variável independente deve ser considerada eticamente e é considerada descritiva, cuja finalidade é observar, descrever e documentar os aspectos da situação, tendendo a ser realista e de difícil crítica(6).

E $m$ relação ao delineamento metodológico faz-se necessário compreender a associação de sua escolha com o objeto a ser estudado e não à preferência do pesquisador ${ }^{(13)}$. O u seja, dependendo do objeto estudado, são identificadas variações sobre 0 delineamento metodológico usado(14).

No cenário das pesquisas qualitativas, a opção por um método e suas técnicas de coleta, o tratamento dos dados, é importante que o pesquisador obtenha uma visão global dos resultados ${ }^{(15)}$.

A tendência temática que mais apareceu nas disser tações e teses foi o levantamento e validação de diagnóstico de enfermagem.

$\mathrm{N}$ as dissertações de mestrado, as etapas do processo de enfermagem totalizaram $91,76 \%$ e nas teses de doutorado, $80,77 \%$. Os achados corroboram com estudo da produção do conhecimento em $S A E$, que verificou que sua operacionalização ainda continua sendo o foco principal das publicações, ao passo que infere-se que o processo de enfermagem no Brasil continua em fase de construção, direcionando o desenvolvimento de estratégias para a prática do enfermeiro em diferentes âmbitos.

Quanto à publicação de artigos, evidenciouse um número pequeno. A credita-se que a característica de baixo percentual de artigos publicados extraídos de disser tações e teses pode ser rela- cionada a publicação em periódicos não indexados, ou mesmo, pela ausência publicação na forma de artigo. Outro fato considerado importante refere-se aos anos de conclusão com maior produção serem recentes e às exigências dos periódicos para a publicação, caracterizando um período por vezes longo de espera entre 0 ano da defesa e o da publicação do artigo. Entretanto, enfatiza-se a exigência pela entrega do comprovante de envio e do artigo por diversos programas de pós-graduação em enfermagem, stricto sensu, no depósito final do estudo, evidenciando a preocupação dos programas com a contribuição para a comunidade científica.

\section{CONCLUSÕES}

0 processo de enfermagem é uma atividade exclusiva do enfermeiro, deve ser aplicada em clientes de todas as instituições de saúde. Entretanto, observa-se dificuldades na prática clínica do enfermeiro, fato que pode ser confirmado com a utilização da temática e da escassa produção científica nacional sobre o tema encontrada e analisada neste estudo. Sendo assim, é de vital importância que os enfermeiros expressem seus interesses desenvolvendo e publicando estudos científicos sobre 0 assunto com trabalhos coerentes com a realidade do sistema de saúde.

Diante da complexidade de variáveis envolvidas no processo de enfermagem e da adequação de diferentes abordagens possíveis, constatou-se 0 uso de diferentes delineamentos metodológicos para a investigação deste objeto de estudo.

V erificou-se, nesta investigação, maior publicação a partir de 2000 , sendo a pesquisa qualitativa mais prevalente. A tendência temática mais evidenciada foi assistência de enfermagem, sendo os assuntos mais abordados o levantamento e validação de diagnóstico de enfer magem e todas as fases do processo.

Portanto, houve a constatação de que o delineamento metodológico qualitativo tem sido amplamente empregado nas publicações da enfermagem. As publicações demonstraram que a tendência temática prevalente é o diagnóstico de enfermagem, utilizando a taxonomia da NANDA como classificação dos mesmos.

Considera-se que existem ainda muitas lacunas na produção de conhecimento sobre o tema, em especial o levantamento da produção do conhecimento sobre o processo de enfermagem. 


\section{REFERÊNCIAS}

1 Fontes CM B, Cruz DA L M . Diagnósticos de enfermagem documentados para pacientes de clínica médica. Rev Esc Enferm USP. 2007;41(3):395-402.

2 Azzolin G M C, Peduzzi M. Processo de trabalho gerencial e processo de enfermagem na perspectiva de docentes de enfermagem. Rev G aúcha E nferm. 2007; 28(4):549-55.

3 H orta W A. Processo de enfer magem. São Paulo: E PU ; 1979.

4 Schneider JF. A pesquisa e as políticas de produção do conhecimento em enfermagem [ editorial]. Rev G aúcha E nferm. 2007;28(2):159.

5 Figueiredo RM, Zem-M ascarenhas SH, Napoleão AA , Camargo A B. Caracterização da produção do conhecimento sobre sistematização da assistência de enfermagem no Brasil. Rev Esc Enferm U SP. 2006;40 (2):299-303.

6 Polit DF, Beck CT, H ungler BP. F undamentos de pesquisa em enfer magem: métodos, avaliação e utilização. 5a ed. Porto Alegre: Artmed; 2004.

7 M arziale M H P, M endes IAC. A enfermagem brasileira na era da informação e do conhecimento. Rev Latino-A m E nfer magem. 2005;13(5):607-8.

8 Conselho Regional de Enfermagem (SP). Reflexão: enfer magem mais crítica e digna. São Paulo; 2002.
9 Conselho Federal de Enfermagem (BR). Resolução COFEn n. 272, de 27 ago 2002: dispõe sobre a sistematização da assistência de enfermagem - SAE - nas instituições de saúde brasileiras [ Internet]. Rio de Janeiro; 2002 [ citado 2005 abr 17] . Disponível em: http:/ / site.portal cofen.gov.br/ node/ 4309.

10 Silva D CN, Ribeiro AA, Fabrício SCC. Produção do conhecimento sobre sistematização da assistência de enfermagem a idosos. Rev E nferm U ERJ. 2007;15 (3):406-10

$11 \mathrm{D}$ emo P. Pesquisa qualitativa: busca de equilíbrio entre for ma e conteúdo. Rev Latino-A m E nfer magem [ I nternet] . 1998 [ citado 2009 jul 30] ;6(2):89-104. Disponível em: www.scielo.br/pdf/ rlae/ v6n2/ 13912.pdf.

12 Oliveira E B, Lisboa M T L. A nálise da produção científica da vertente saúde do trabalhador de enfermagem: subjetividade etrabalho. Rev Enferm U ERJ. 2004;12(1):24-9.

13 L oBiondo-W ood G, H aber J. Pesquisa em Enfermagem: métodos, avaliação crítica e utilização. 4⿳亠丷 ed. Rio de Janeiro: G uanabara Koogan; 2001.

14 A Imeida VCF, Damasceno M M C, A raújo T L . Saúde do trabalhador de saúde: análise sobre o tema. Rev Bras Enferm. 2005;58(3):335-40.

15 Campos CJ G, T urato ER. A nálise de conteúdo em pesquisas que utilizam metodologia clínico-qualitativa: aplicação e perspectivas. Rev Latino-Am Enfermagem. 2009;17(2):259-64.

\section{Endereço da autora / Dirección del autor / Author's address:}

Erika Christiane M arocco Duran

Rua T essália Vieira de Camargo, 126,

Cidade U niver sitária "Z eferino Vaz"

13084-971, Campinas, SP

E-mail: ecduran@fcm.unicamp.br
Recebido em: 10/ 09/2010

A provado em: 25/ 05/ 2011 